\title{
Study on the Application of Microwave Plasma Technology in the Desulfurization Process
}

\author{
YuQiong $\mathrm{Wu}^{1, \mathrm{a}}$ \\ ${ }^{1}$ Hubei Key Laboratory of Industrial Fume and Dust Pollution Control, Jianghan University, HuBei, \\ Wuhan, 430056, China \\ a99067410@qq.com
}

Keywords: Microwave; Plasma; Sulfur dioxide; Desulfurization rate

Abstract. With sulfur dioxide and other environmental pollution is becoming more and more serious. It is of great significance to explore an efficient, fast and clean way to remove these environmental pollutants. In this paper, the microwave plasma technology is applied in the desulfurization process. The effect of microwave power, carrier gas, carrier gas type and sulfur dioxide concentration on the desulfurization efficiency is studied. The results show that: in the microwave power $60 \mathrm{~W}$, the oxygen carrier gas flow rate of $0.8 \mathrm{~L} / \mathrm{min}, \mathrm{SO}_{2}$ intake concentration of $20 \mathrm{mg} / \mathrm{m}^{3}$, the desulfurization rate can reach $98.6 \%$. In the management of air pollution, this paper proves that microwave plasma technology is one of the most promising and effective technical methods.

\section{Introduction}

$\mathrm{SO}_{2}$ is the main cause of the formation of acid rain; its pollution has a low concentration, wide range, long time characteristics. Its hazard is chronic and cumulative [1]. In addition, the results of animal experiments also show that $\mathrm{SO}_{2}$ is a tumor promoting [2]. Therefore, it is urgent to treatment of acid rain pollution and control the emission of $\mathrm{SO}_{2}$ [3].

At present, the treatment methods of $\mathrm{SO}_{2}$ mainly include physical method, biological method [4] and chemical method [5] and so on. However, the above methods have their own limitations, large-scale industrial applications are limited. Plasma desulfurization technology as a new desulfurization technology is constantly developing and improving. Low temperature plasma technology to deal with $\mathrm{SO}_{2}$ has the advantages of low energy consumption, high efficiency, less equipment investment and so on [6]. Therefore, the treatment efficiency of sulfur dioxide is significantly improved by using plasma technology. With the deep discussion on the reaction mechanism of the plasma technology, people begin to focus on product selectivity; the purpose is to reduce the types and quantities of by-products, while reducing the secondary pollution as much as possible [7]. In this paper, we explore the use of microwave plasma technology for desulfurization process.

\section{Materials and Equipment}

Materials and Reagents. Microwave plasma device (homemade); rotor flowmeter LZB-2 Yuyao Yinhuan flowmeter Instrument Co. Ltd; argon Wuhan Tianci Gas Co., Ltd.; oxygen Wuhan Tianci Gas Co., Ltd.; sulfur dioxide Wuhan newradar Trade Co., Ltd.; $\mathrm{SO}_{2}$ pump suction detection instrument Shenzhen Peng Lei Technology Co., Ltd.

Experimental Technique. The process schematic diagram is shown in Fig. 1. The microwave plasma device utilizes the microwave to excite the thin gas, thereby producing the plasma. Cylinder gas flow is controlled by the rotor flowmeter, to strictly ensure the component proportion of mixed gas; mixed gas is sent to the plasma reactor for desulfurization reaction; the reaction gas is fed into a sulfur dioxide analyzer to determine the change in its composition. According to the analysis results, the removal rate of sulfur dioxide can be calculated. The calculation formula is as follows: 


$$
X_{\mathrm{SO}_{2}}=\left(1-\frac{c_{\mathrm{SO}_{2}}}{c_{\mathrm{SO}_{2}}^{0}}\right) \times 100 \%
$$

$c_{\mathrm{SO}_{2}}$ - The amount of sulfur dioxide after the reaction $c_{\mathrm{SO}_{2}}^{0}$-The amount of sulfur dioxide before the reaction.

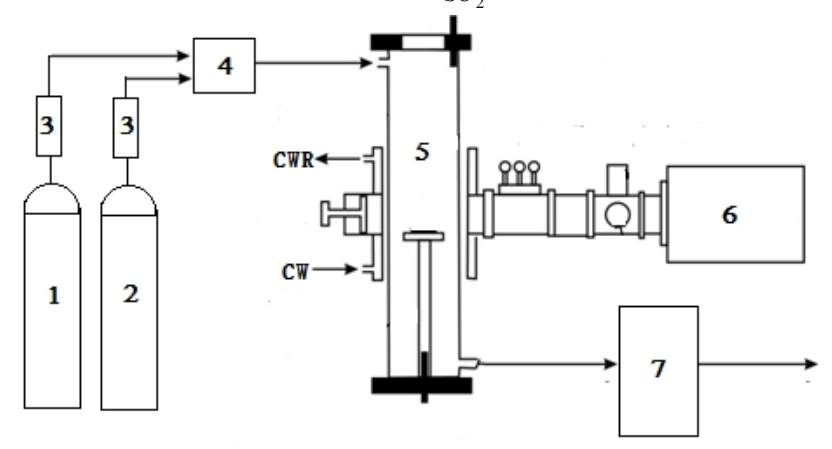

1. Sulfur Dioxide 2. Carrier Gas 3. Rotor Flow Meter 4. Mixer

5. Microwave Plasma Equipment 6. Microwave Generator 7. $\mathrm{SO}_{2}$ Analyzer

Fig. 1 Schematic diagram of process

Reaction Mechanism. Under the plasma condition, it is possible to remove the $\mathrm{SO}_{2}$ from the two ways of oxidation and reduction [8]. As the carrier gas accounts for the largest component, which absorbs most of the energy of the plasma discharge, and generates excited state molecules. The collision between the particles and $\mathrm{SO} 2$ molecules, it is possible to make $\mathrm{SO} 2$ dissociation and generate sulfur. Another way is the oxidation process. Under the action of plasma, $\mathrm{O}_{2}$ is added to generate sufficient oxidizing particles, such as $\mathrm{O}, \mathrm{O}_{3}, \mathrm{O}_{2}{ }^{*}$, etc., $\mathrm{SO}_{2}$ may be in the role of these particles to remove oxidation.

\section{Results and discussion}

The Effect of Microwave Power on the Desulfurization Rate. The carrier gas flow rate is $0.5 \mathrm{~L} / \mathrm{min}$, when the concentration is constant; the effect of microwave power on the desulfurization rate is shown in Fig. 2. The shape and the emission intensity of the plasma are obviously influenced by the microwave power in the experiment. Microwave power is too small, the formation of the plasma flame is small and unstable; the microwave power increases, the plasma is more stable, and easy to form a clear plasma flame.

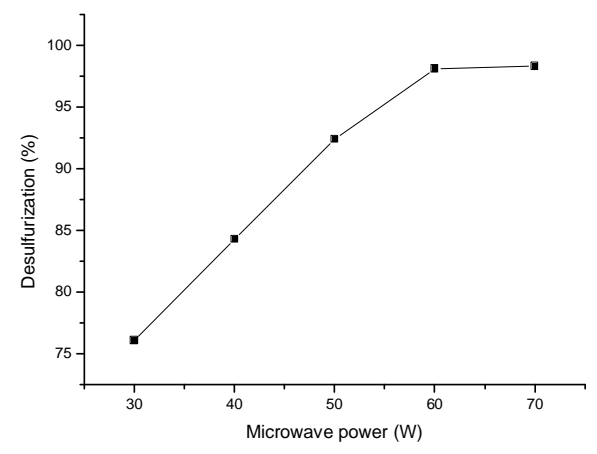

Fig. 2 Effect of microwave power on the rate

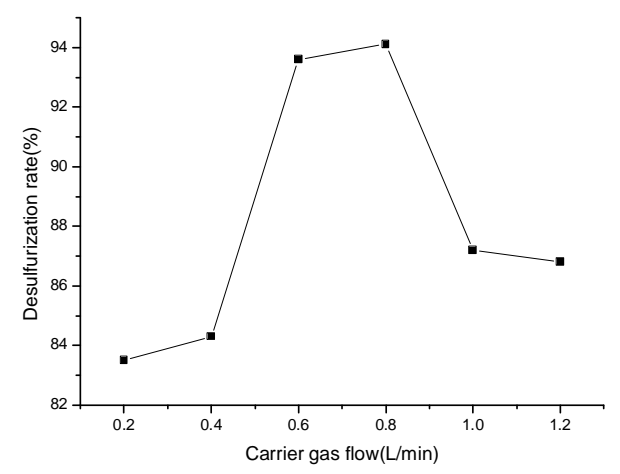

Fig. 3 Effect of gas content on desulfurization desulfurization rate

As shown in Fig. 2, the emission intensity increases with the increase of microwave power, the volume of plasma increases, and the dissociation ability and excitation capacity of sulfur dioxide are obviously enhanced. That is to say, with the increase of microwave power, the desulfurization rate is also higher, 
the power of $30 \sim 60 \mathrm{~W}$, the desulfurization rate increased faster, when the microwave power is greater than $60 \mathrm{~W}$, plasma torch will no longer increases with the power increasing, sulfur dioxide removal rate is stabilized. In order to ensure the stability and sensitivity of the instrument, microwave power is selected 60W.

Effect of Gas Content on Desulfurization Rate. Effect of different gas loading on desulfurization rate is shown in Fig. 3.

The effects of carrier gas on the removal rate of sulfur dioxide showed a trend of S type, that under the low flow, desulfurization rate increases with the carrier gas increased; when the volume of carrier gas is too small (less than $0.6 \mathrm{~L} / \mathrm{min}$ ), the torch can not form cross point, that is not conducive to the reaction of the dissociation; when the carrier gas volume is too large (higher than $0.9 \mathrm{~L} / \mathrm{min}$ ) and $\mathrm{SO}_{2}$ molecules on the plasma equipment in residence time reduced, the plasma flame instability and noise also significantly increased. Comprehensive consideration, the selection of carrier gas volume is about $0.8 \mathrm{~L} / \mathrm{min}$, the effect of plasma reaction is obvious, and the desulfurization rate is the highest.

Effect of Carrier Gas Type on Desulfurization Rate. Under certain conditions, the use of carrier gas type is different, and the color of the plasma flame will be changed obviously. When the carrier gas is air, the flame is bright yellow; when the carrier gas is oxygen, the flame color is dark, close to the transparent color; when the carrier gas is argon, the flame color is blue.

Under the condition of constant microwave power, the oxygen and argon gas is used for the degradation of sulfur dioxide, as shown in Fig. 4. As shown in Fig. 4, there is a slight difference in the effect of different carrier gas on degradation. With the increase of sulfur dioxide concentration, the degradation effect of oxygen is slightly better. The main reason for this result may be due to the involvement of oxygen in the plasma reaction, so that the gas molecules are degraded more thoroughly. So oxygen is chosen as carrier gas.

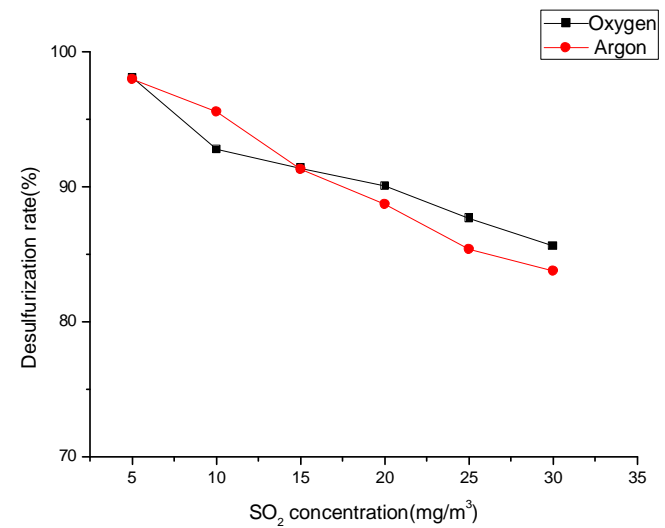

Fig. 4 Effect of carrier gas type on desulfurization rate

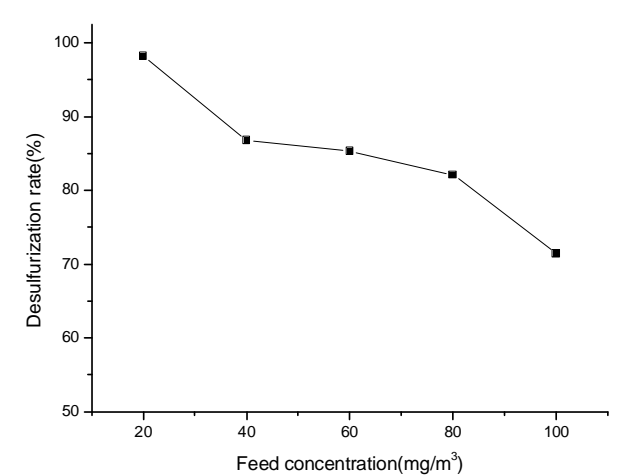

Fig. 5 Effect of sulfur dioxide concentration on desulfurization rate

Effect of Sulfur Dioxide Concentration on Desulfurization Rate. Under the condition of microwave power and oxygen content, the effect of initial concentration of sulfur dioxide on the desulfurization rate was investigated. As shown in Fig. 5.

As shown in Fig. 5, with the increase in the concentration of sulfur dioxide, the desulfurization rate is gradually decreased. When the sample concentration is below $20 \mathrm{mg} / \mathrm{m}^{3}$, the desulfurization rate is $98.2 \%$, but when the initial concentration is $100 \mathrm{mg} / \mathrm{m}^{3}$, the desulfurization rate is reduced to $71.4 \%$. While in the low concentration of sulfur dioxide, the desulfurization rate is higher, but with the increase of the concentration of sulfur dioxide, the absolute amount of desulfurization is increased. This analysis shows that content of sulfur dioxide concentration does not change experiment microwave discharge characteristics of plasma, when $\mathrm{SO}_{2}$ molecule is less, the number of electrons and the free base more, the low concentration $\mathrm{SO}_{2}$ has higher removal rate. When the discharge condition is constant, the concentration of the active particles and the excited state molecules are approximately the same. To deal with the high concentration of pollutants, the concentration of the active groups and excited state 
molecules is also high. When the concentration of $\mathrm{SO}_{2}$ is too high, the microwave discharge can not provide enough active particles.

\section{Verification Experiment}

In the microwave power $60 \mathrm{~W}$, oxygen carrier gas volume $0.8 \mathrm{~L} / \mathrm{min}, \mathrm{SO}_{2}$ air intake concentration $20 \mathrm{mg} / \mathrm{m}^{3}$, three sets of parallel experiments are done; the desulfurization rate can reach $98.6 \%$.

\section{Conclusions}

The effect of microwave power, gas content, type of carrier gas and sulfur dioxide concentration on the desulfurization rate is studied by using microwave plasma technology. The results show that with the increase of microwave power, the desulfurization rate increases gradually. When the microwave power is higher, the noise of the detector and the reflected current increase significantly, which is not conducive to the degradation of sulfur dioxide. Therefore, the microwave power is generally selected $60 \mathrm{~W}$; carrier gas volume is an important factor affecting the degradation effect, the size of the $\mathrm{SO}_{2}$ in the plasma sphere of residence time, but also affects the shape and stability of the plasma. The carrier gas volume is too large or too small will lead to the low rate of desulfurization, and carrier gas flow rate is chosen to be at $0.81 / \mathrm{min}$; type of carrier gas on desulfurization rate has little effect, oxygen is used as the carrier gas, whose effect is slightly better than that of argon, so oxygen is selected as the carrier gas; sampling initial concentration influence the plasma reaction ability size, when the flow rate is low, the plasma ball is stable, and larger and concentrated, $\mathrm{SO}_{2}$ by the degree of ionization is more complete, high degradation rate and $\mathrm{SO}_{2}$ inlet concentration is chosen to be $20 \mathrm{mg} / \mathrm{m} 3$. Through the verification test, the optimal process conditions are obtained: microwave power $60 \mathrm{~W}$, carrier gas flow rate $0.8 \mathrm{~L} / \mathrm{min}, \mathrm{SO}_{2}$ intake concentration $20 \mathrm{mg} / \mathrm{m}^{3}$, the desulfurization rate can reach $98.6 \%$.

Microwave plasma technology as a new kind of desulfurization technology is constantly developing and improving. It is the use of extreme physical means to solve some problems in the desulfurization process. Compared to the traditional flue gas desulfurization technology, it has a wider range of application, higher purification efficiency, faster response and lower operating costs. It is the most potential and promising technology.

\section{Acknowledgements}

In this paper, the research was sponsored by Jianghan University graduate course construction project in 2014 (Project No. 04200021).

\section{References}

[1] J.M. Hao. Handbook of Sulfur Dioxide Pollution Control in Coal Combustion. Chemical Industry Press(2001)

[2] F.Y. Mou. Effect on the Health of Workers Exposed to Low-Concentration Sulphur Dioxide in Long Term, Preventive Medicine Tribune Vol. 11 (2005), p. 298-299

[3] Gonzalez-sanchez A, Revah S, Deshusses Marc A . Alkaline Biofihralion of $\mathrm{H}_{2} \mathrm{~S}$ odors . Environ Sci Technol Vol. 42 (2008), p. 7398-7404

[4] K.B. Wu. Construction Project on Status Quo of Odor Pollution and Control, Straits Science Vol. 8 (2008), p. $17-22$

[5] N.Q. Y , Z. Qu . Removal Characteristics of Gaseous Sulfurcontaining Compounds by Pulsed Corona Plasma, Ind Eng Chem Res Vol. 45 (2006), p.6420-6427 
[6] Li Y. Zhen, Y.B. Sun, J.W. Xu, J.H. Feng, J.Y. Wang, H.Y. Wang, Y. Chen, H. Miu . Research Status of Odors Decomposition by Non - Thermal Plasma (NTP), Electric power technology and environmental protection Vol. 26 (2010), p. 58-62

[7] Lu Changyi. Introduction to Modern Environmental Science. Xiamen University press(2005)

[8] B. Sun, X.X. Pan, J. Hou, W.M. Sun, H.Q. Hou , Study on Plasma Dissociation of $\mathrm{SO}_{2}$ and its Reaction Path, Journal of Fudan University (Natural Science) Vol. 40 (2001), p. 411-415 